\title{
On the identifiability testing in blind source separation using resampling technique
}

\author{
A. Aïssa-El-Bey, K. Abed-Meraim and Y. Grenier \\ ENST, TSI department, 46 rue Barrault 75634, Paris Cedex 13, France \\ \{elbey, abed, grenier\}@tsi.enst.fr
}

\begin{abstract}
This paper focuses on the second order identifiability problem of blind source separation and its testing. We present first necessary and sufficient conditions for the identifiability and partial identifiability using a finite set of correlation matrices. These conditions depend on the autocorrelation fonction of the unknown sources. However, it is shown here that they can be tested directly from the observation through the decorrelator output. This issue is of prime importance to decide whether the sources have been well separated or else if further treatments are needed. We then propose an identifiability testing based on resampling (jackknife) technique, that is validated by simulation results.
\end{abstract}

\section{Introduction}

Blind source separation (BSS) of instantaneous mixtures has attracted so far a lot of attention due to its many potential applications [1] and its mathematical tractability that lead to several nice and simple BSS solutions $[1,2,5,13]$. The underlaying model is given by:

$$
\mathbf{x}(t)=\mathbf{y}(t)+\mathbf{w}(t)=\mathbf{A} \mathbf{s}(t)+\mathbf{w}(t)
$$

where $\mathbf{s}(t)=\left[s_{1}(t), \cdots, s_{m}(t)\right]^{T}$ is the $m \times 1$ complex source vector, $\mathbf{w}(t)=$ $\left[w_{1}(t), \cdots\right.$,

$\left.w_{n}(t)\right]^{T}$ is the $n \times 1$ complex noise vector, $\mathbf{A}$ is the $n \times m$ full column rank mixing matrix (i.e., $n \geq m$ ), and the superscript $T$ denotes the transpose operator. The source signal vector $\mathbf{s}(t)$, is assumed to be a multivariate stationary complex stochastic process.

In this paper we consider only the second order BSS methods and hence the component processes $s_{i}(t), 1 \leq i \leq m$ are assumed to be temporally coherent and mutually uncorrelated, with zero mean and second order moments:

$$
\mathbf{S}(\tau) \stackrel{\text { def }}{=} E\left(\mathbf{s}(t+\tau) \mathbf{s}^{\star}(t)\right)=\operatorname{diag}\left[\rho_{1}(\tau), \cdots, \rho_{m}(\tau)\right]
$$

where $\rho_{i}(\tau) \stackrel{\text { def }}{=} E\left(s_{i}(t+\tau) s_{i}^{*}(t)\right)$, the expectation operator is $E$, and the superscripts $*$ and $\star$ denote the conjugate of a complex number and the complex conjugate transpose of a vector, respectively. The additive noise $\mathbf{w}(t)$ is modeled as a white stationary zero-mean complex random process. In that case, the 
source separation is achieved by decorrelating the signals at different time lags. This is made possible under certain identifiability conditions that have been developed in [3] and recalled briefly in this paper.

Although the previous conditions are expressed in terms of the autocorrelation coefficient of the unknown source signals, we propose here a solution to test them directly out of the received data using the jackknife (resampling) technique.

\section{Second Order Identifiability}

In [4], Tong et al. have shown that the sources are blindly separable based on (the whole set) of second order statistics only if they have different spectral density functions. In practice we achieve the BSS using only a finite set of correlation matrices. Therefore, the preview identifiability result was generalized to that case in $[5,3]$ leading to the necessary and sufficient identifiability conditions given by the following theorem:

Theorem 1. Let $\tau_{1}<\tau_{2}<\cdots<\tau_{K}$ be $K \geq 1$ time lags, and define $\boldsymbol{\rho}_{i}=$ $\left[\rho_{i}\left(\tau_{1}\right), \rho_{i}\left(\tau_{2}\right)\right.$,

$\left.\cdots, \rho_{i}\left(\tau_{K}\right)\right]$ and $\tilde{\boldsymbol{\rho}}_{i}=\left[\Re\left(\boldsymbol{\rho}_{i}\right), \Im\left(\boldsymbol{\rho}_{i}\right)\right]$ where $\Re(x)$ and $\Im(x)$ denote the real part and imaginary part of $x$, respectively. Taking advantage of the indetermination, we assume without loss of generality that the sources are scaled such that $\left\|\boldsymbol{\rho}_{i}\right\|=$ $\left\|\tilde{\boldsymbol{\rho}}_{i}\right\|=1$, for all $i^{1}$. Then, BSS can be achieved using the output correlation matrices at time lags $\tau_{1}, \tau_{2}, \cdots, \tau_{K}$ if and only if for all $1 \leq i \neq j \leq m$ :

$$
\tilde{\boldsymbol{\rho}}_{i} \text { and } \tilde{\boldsymbol{\rho}}_{j} \text { are (pairwise) linearly independent }
$$

Interestingly, we can see from condition (1) that BSS can be achieved from only one correlation matrix $\mathbf{R}_{x}(k) \stackrel{\text { def }}{=} E\left(\mathbf{x}(t+k) \mathbf{x}^{\star}(t)\right)$ provided that the vectors $\left[\Re\left(\rho_{i}(k)\right), \Im\left(\rho_{i}(k)\right]\right.$ and $\left[\Re\left(\rho_{j}(k)\right), \Im\left(\rho_{j}(k)\right]\right.$ are pairwise linearly independent for all $i \neq j$.

Note also that, from (1), BSS can be achieved if at most one temporally white source signal exists. In contrast, recall that when using higher order statistics, BSS can only be achieved if at most one Gaussian source signal exists.

Under the condition of Theorem 1, the BSS can be achieved by decorrelation according to the following result:

Theorem 2. Let $\tau_{1}, \tau_{2}, \cdots, \tau_{K}$ be $K$ time lags and $\mathbf{z}(t)=\left[z_{1}(t), \cdots, z_{m}(t)\right]^{T}$ be an $m \times 1$ vector given by $\mathbf{z}(t)=\mathbf{B} \mathbf{x}(t)$. Define $r_{i j}(k) \stackrel{\text { def }}{=} E\left(z_{i}(t+k) z_{j}^{*}(t)\right)$. If the identifiability condition holds, then $\mathbf{B}$ is a separating matrix (i.e. $\mathbf{B y}(t)=$ $\mathbf{P} \Lambda \mathbf{s}(t)$ for a given permutation matrix $\mathbf{P}$ and a non-singular diagonal matrix A) if and only if

$$
r_{i j}(k)=0 \quad \text { and } \quad \sum_{k=\tau_{1}}^{\tau_{K}}\left|r_{i i}(k)\right|>0
$$

\footnotetext{
${ }^{1}$ We implicitly assume here that $\boldsymbol{\rho}_{i} \neq 0$, otherwise the source signal could not be detected (and a fortiori could not be estimated) from the considered set of correlation matrices. This hypothesis will be held in the sequel.
} 
for all $1 \leq i \neq j \leq m$ and $k=\tau_{1}, \tau_{2}, \cdots, \tau_{K}$.

Note that, if one of the time lags is zero, the result of Theorem 2 holds only under the noiseless assumption. In that case, we can replace the condition $\sum_{k=\tau_{1}}^{\tau_{K}}\left|r_{i i}(k)\right|>0$ by $r_{i i}(0)>0$, for $i=1, \cdots, m$. On the other hand, if all the time lags are non-zero and if the noise is temporally white (but can be spatially colored with unknown spatial covariance matrix) then the above result holds without the noiseless assumption.

Based on Theorem 2, we can define different objective (contrast) functions for signal decorrelation. In [6], the following criterion ${ }^{2}$ was used

$$
G(\mathbf{z})=\sum_{k=\tau_{1}}^{\tau_{K}} \log \left|\operatorname{diag}\left(\mathbf{R}_{z}(k)\right)\right|-\log \left|\mathbf{R}_{z}(k)\right|
$$

where $\operatorname{diag}(\mathbf{A})$ is the diagonal matrix obtained by zeroing the off diagonal entries of $\mathbf{A}$. Another criterion used in [7] is

$$
G(\mathbf{z})=\sum_{k=\tau_{1}}^{\tau_{K}} \sum_{1 \leq i<j \leq m}\left|r_{i j}(k)\right|^{2}+\sum_{i=1}^{m}\left|\sum_{k=\tau_{1}}^{\tau_{K}}\right| r_{i i}(k)|-1|^{2}
$$

Equations (3) and (4) are non-negative functions which are zero if and only if $\mathbf{R}_{z}(k)=E\left(\mathbf{z}(n+k) \mathbf{z}^{\star}(n)\right)$ are diagonal for $k=\tau_{1}, \cdots, \tau_{K}$ or equivalently if (2) is met.

\section{Partial Identifiability}

It is generally believed that when the identifiability conditions are not met, the BSS cannot be achieved. This is only half of the truth as it is possible to partially separate the sources in the sense that we can extract those which satisfy the identifiability conditions. More precisely, the sources can be separated in blocks each of them containing a mixture of sources that are not separable using the considered set of statistics. For example, consider a mixture of 3 sources such that $\tilde{\boldsymbol{\rho}}_{1}=\tilde{\boldsymbol{\rho}}_{2}$ while $\tilde{\boldsymbol{\rho}}_{1}$ and $\tilde{\boldsymbol{\rho}}_{3}$ are linearly independent. In that case, source $s_{3}$ can be extracted while sources $s_{1}$ and $s_{2}$ cannot. In other words, by decorrelating the observed signal at the considered time lags, one obtain 3 signals one of them being $s_{3}$ (up to a scalar constant) and the two others are linear mixtures of $s_{1}$ and $s_{2}$.

This result can be mathematically formulated as follows: assume there are $d$ distinct groups of sources each of them containing $d_{i}$ source signals with same (up to a sign) correlation vector $\tilde{\boldsymbol{\rho}}_{i}, i=1, \cdots, d$ (clearly, $m=d_{1}+\cdots+d_{d}$ ). The correlation vectors $\tilde{\boldsymbol{\rho}}_{1}, \cdots, \tilde{\boldsymbol{\rho}}_{d}$ are pairwise linearly independent. We write $\mathbf{s}(t)=\left[\mathbf{s}_{1}^{T}(t), \cdots, \mathbf{s}_{d}^{T}(t)\right]^{T}$ where each sub-vector $\mathbf{s}_{i}(t)$ contains the $d_{i}$ source signals with correlation vector $\tilde{\boldsymbol{\rho}}_{i}$.

\footnotetext{
${ }^{2}$ In that paper, only the case where $\tau_{1}=0$ was considered.
} 
Theorem 3. Let $\mathbf{z}(t)=\mathbf{B} \mathbf{x}(t)$ be an $m \times 1$ random vector satisfying equation (2) for all $1 \leq i \neq j \leq m$ and $k=\tau_{1}, \cdots, \tau_{K}$. Then, there exists a permutation matrix $\mathbf{P}$ such that $\overline{\mathbf{z}}(t) \stackrel{\text { def }}{=} \mathbf{P} \mathbf{z}(t)=\left[\overline{\mathbf{z}}_{1}^{T}(t), \cdots, \overline{\mathbf{z}}_{d}^{T}(t)\right]^{T}$ where $\overline{\mathbf{z}}_{i}(t)=\mathbf{U}_{i} \mathbf{s}_{i}(t)$, $\mathbf{U}_{i}$ being a $d_{i} \times d_{i}$ non-singular matrix. Moreover, sources belonging to the same group, i.e., having same (up to a sign) correlation vector $\tilde{\boldsymbol{\rho}}_{i}$ can not be separated using only the correlation matrices $\mathbf{R}_{x}(k), k=\tau_{1}, \cdots, \tau_{K}$.

This result (see [3])shows that when some of the sources have same (up to a sign) correlation vectors then the best that can be done is to separate them per blocks and this can be achieved by decorrelation. However, this result would be useless if we cannot check the linear dependency of the correlation vectors $\tilde{\boldsymbol{\rho}}_{i}$ and partition the signals per groups (as shown above) according to their correlation vectors. This leads us to the important problem of testing the identifiability condition that is discussed next.

\section{Testing of identifiability condition}

\subsection{Theoretical result}

The necessary and sufficient identifiability condition (1) depends on the correlation coefficients of the source signals. The latter being unknown, it is therefore impossible to a priori check whether the sources are 'separable' or not from a given set of output correlation matrices. However, it is possible to check a posteriori whether the sources have been 'separated' or not. We have the following result [3]:

Theorem 4. Let $\tau_{1}<\tau_{2}<\cdots<\tau_{K}$ be $K$ distinct time lags and $\mathbf{z}(t)=\mathbf{B} \mathbf{x}(t)$. Assume that $\mathbf{B}$ is a matrix such that $\mathbf{z}(t)$ satisfies ${ }^{3}$ equation (2) for all $1 \leq i \neq$ $j \leq m$ and $k=\tau_{1}, \cdots, \tau_{K}$. Then there exists a permutation matrix $\mathbf{P}$ such that for $k=\tau_{1}, \cdots, \tau_{K}$.

$$
E\left(\mathbf{z}(t+k) \mathbf{z}^{\star}(t)\right)=\mathbf{P}^{T} \mathbf{S}(k) \mathbf{P}
$$

In other words the entries of $\overline{\mathbf{z}}(t) \stackrel{\text { def }}{=} \mathbf{P} \mathbf{z}(t)$ have the same correlation coefficients as those of $\mathbf{s}(t)$ at time lags $\tau_{1}, \cdots, \tau_{K}$, i.e. $E\left(\bar{z}_{i}(t+k) \bar{z}_{i}^{*}(t)\right)=\rho_{i}(k)$ for $k=$ $\tau_{1}, \cdots, \tau_{K}$ and $i=1, \cdots, m$.

From Theorem 4, the existence of condition (1) can be checked by using the approximate correlation coefficients $r_{i i}(k) \stackrel{\text { def }}{=} E\left(z_{i}(t+k) z_{i}^{\star}(t)\right)$. It is important to point out that even if equation (2) holds, it does not mean that the source signals have been separated. Three situations may happen:

1. For all pairs $(i, j), \tilde{\boldsymbol{\rho}}_{i}$ and $\tilde{\boldsymbol{\rho}}_{j}$ (computed from $\mathbf{z}(t)$ ) are pairwise linearly independent. Then we are sure that the sources have been separated and that $\mathbf{z}(t)=\mathbf{s}(t)$ up to the inherent indeterminacies of the BSS problem.

\footnotetext{
${ }^{3}$ Because of the inherent indetermination of the BSS problem, we assume without loss of generality that the exact and estimated sources are similarly scaled, i.e., $\left\|\tilde{\boldsymbol{\rho}}_{i}\right\|=1$.
} 
In fact, the testing of the identifiability condition is equivalent to pairwise testing the angles between $\tilde{\boldsymbol{\rho}}_{i}$ and $\tilde{\boldsymbol{\rho}}_{j}$ for all $1 \leq i \neq j \leq m$. The larger the angle between $\tilde{\boldsymbol{\rho}}_{i}$ and $\tilde{\boldsymbol{\rho}}_{j}$, the better the quality of source separation (see performance analysis in $[5])$.

2. For all pairs $(i, j), \tilde{\boldsymbol{\rho}}_{i}$ and $\tilde{\boldsymbol{\rho}}_{j}$ are linearly dependent. Thus the sources haven't been separated and $\mathbf{z}(t)$ is still a linear combination of $\mathbf{s}(t)$.

3. A few pairs $(i, j)$ out of all pairs satisfy $\tilde{\boldsymbol{\rho}}_{i}$ and $\tilde{\boldsymbol{\rho}}_{j}$ linearly dependent. Therefore the sources have been separated in blocks.

Now, having only one signal realization at hand, we propose to use a resampling technique to evaluate the statistics needed for the testing.

\subsection{Testing using resampling techniques}

Note that in practice the source correlation coefficients are calculated from noisy finite sample data. Due to the joint effects of noise and finite sample size, it is impossible to obtain the exact source correlation coefficients to test the identifiability condition. The identifiability condition should be tested using a certain threshold $\alpha$, i.e., decide that $\tilde{\boldsymbol{\rho}}_{i}$ and $\tilde{\boldsymbol{\rho}}_{j}$ are linearly independent if ||$\tilde{\boldsymbol{\rho}}_{i} \tilde{\boldsymbol{\rho}}_{j}^{T}|-1|>\alpha$. To find $\alpha$ we use the fact that the estimation error of $\tilde{\boldsymbol{\rho}}_{i} \tilde{\boldsymbol{\rho}}_{j}^{T}$ is asymptotically gaussian ${ }^{4}$ and hence one can build the confidence interval of such a variable according to its variance. This algorithm can be summarized as follows:

1. Estimate a demixing matrix $\mathbf{B}$ and $\mathbf{z}(t) \stackrel{\text { def }}{=} \mathbf{B x}(t)$ using an existing second order decorrelation algorithm (e.g. SOBI [5]).

2. For each component $z_{i}(t)$, estimate the corresponding normalized vector $\widetilde{\boldsymbol{\rho}}_{i}$.

3. Calculate the scalar product $\hat{R}(i, j)=\left|\tilde{\boldsymbol{\rho}}_{i} \tilde{\boldsymbol{\rho}}_{j}^{T}\right|$ for each pair $(i, j)$.

4. Estimate $\hat{\sigma}_{(i, j)}$ the standard deviation of $\hat{R}(i, j)$ using resampling technique (see Section 5).

5. Choose $\alpha_{(i, j)}$ according to the confidence interval. e.g. to have a confidence interval equal to $99.7 \%$ we choose $\alpha_{(i, j)}=3 \hat{\sigma}_{(i, j)}$, and compare $|\hat{R}(i, j)-1|$ to $\alpha_{(i, j)}$ to test whether sources $i$ and $j$ have been separated or not.

\section{Resampling techniques: The jackknife}

In many signal processing applications one is interested in forming estimates of a certain number of unknown parameters of a random process, using a set of sample values. Further, one is interested in finding the sampling distribution of the estimators, so that the respective means, variances, and cumulants can be calculated, or in making some kind of probability statements with respect to the unknown true values of the parameters.

The bootstrap [8] was introduced by Efron [9] as an approach to calculate confidence intervals for parameters in circumstances where standard methods cannot

\footnotetext{
${ }^{4}$ More precisely, one can prove that the estimation error $\sqrt{T} \delta\left(\tilde{\boldsymbol{\rho}}_{i} \tilde{\boldsymbol{\rho}}_{j}^{T}\right)$ is asymptotically, i.e. for large sample size $T$, gaussian with zero mean and finite variance.
} 
be applied. The bootstrap has subsequently been used to solve many other problems that would be too complicated for traditional statistical analysis.

In simple words, the bootstrap does with a computer what the experimenter would do in practice, i.e. if it were possible: he or she would repeat the experiment. With the bootstrap, the observations are randomly reassigned, and the estimates recomputed. These assignments and recomputations are done hundreds or thousands of times and treated as repeated experiments.

The jackknife [10] is another resampling technique for estimating the standard deviation. As an alternative to the bootstrap, the jackknife method can be thought of as drawing $n$ samples of size $n-1$ each without replacement from the original sample of size $n$ [10].

Suppose we are given the sample $\mathcal{X}=\left\{X_{1}, X_{2}, \ldots, X_{n}\right\}$ and an estimate, $\hat{\vartheta}$, from $\mathcal{X}$. The jackknife method is based on the sample delete-one observation at a time,

$$
\mathcal{X}^{(i)}=\left\{X_{1}, X_{2}, \ldots, X_{i-1}, X_{i+1}, \ldots, X_{n}\right\}
$$

for $i=1,2, \ldots, n$, called the jackknife sample. This $i^{\text {th }}$ jackknife sample consists of the data set with the $i^{\text {th }}$ observation removed. For each $i^{\text {th }}$ jackknife sample, we calculate the $i^{t h}$ jackknife estimate, $\hat{\vartheta}^{(i)}$ of $\vartheta, i=1,2, \ldots, n$. The jackknife estimate of the standard deviation of $\hat{\vartheta}$ is defined by

$$
\hat{\sigma}=\sqrt{\frac{n-1}{n} \sum_{i=1}^{n}\left(\hat{\vartheta}^{(i)}-\frac{1}{n} \sum_{j=1}^{n} \hat{\vartheta}^{(j)}\right)^{2}}
$$

The jackknife is computationally less expensive if $n$ is less than the number of replicates used by the bootstrap for standard deviation estimation because it requires computation of $\hat{\vartheta}$ only for the $n$ jackknife data sets. For example, if $L=25$ resamples are necessary for standard deviation estimation with the bootstrap, and the sample size is $n=10$, then clearly the jackknife would be computationally less expensive than the bootstrap. In order to test the separability of the estimated signals, we have used a jackknife method to estimate the variance of the scalar product quantities $R(i, j)$ for $i, j=1,2, \ldots, m$. This is done according to the following steps:

1. From each signal $\mathbf{z}_{i}=\left[z_{i}(0), \ldots, z_{i}(T-1)\right]^{T}$, generate $T$ vectors such as $\mathbf{z}_{i}^{(j)}=\left[z_{i}(0), \ldots, z_{i}(j-1), z_{i}(j+1), \ldots, z_{i}(T-1)\right]^{T}$ and $j=0,1, \ldots, T-1$.

2. For each vector $\mathbf{z}_{i}^{(j)}$, estimate the corresponding vector $\widetilde{\boldsymbol{\rho}}_{i}^{(j)}$.

3. Estimate $\hat{\mathbf{R}}$ such as its $(i, j)^{t h}$ entry is

$$
\hat{R}(i, j)=\frac{1}{T} \sum_{k=0}^{T-1} \frac{\left\langle\widetilde{\boldsymbol{\rho}}_{i}^{(k)}, \widetilde{\boldsymbol{\rho}}_{j}^{(k)}\right\rangle}{\left\|\widetilde{\boldsymbol{\rho}}_{i}^{(k)}\right\|\left\|\widetilde{\boldsymbol{\rho}}_{j}^{(k)}\right\|}
$$

where $\langle\cdot, \cdot\rangle$ denotes the scalar product and $\|\cdot\|$ is the euclidian norm. 
4. Estimate the standard deviation of $\hat{R}(i, j)$ by

$$
\hat{\sigma}_{(i, j)}=\sqrt{\frac{T-1}{T} \sum_{k=0}^{T-1}\left(\frac{\left\langle\tilde{\boldsymbol{\rho}}_{i}^{(k)}, \tilde{\boldsymbol{\rho}}_{j}^{(k)}\right\rangle}{\left\|\tilde{\boldsymbol{\rho}}_{i}^{(k)}\right\|\left\|\tilde{\boldsymbol{\rho}}_{j}^{(k)}\right\|}-\frac{1}{T} \sum_{l=0}^{T-1} \frac{\left\langle\tilde{\boldsymbol{\rho}}_{i}^{(l)}, \tilde{\boldsymbol{\rho}}_{j}^{(l)}\right\rangle}{\left\|\tilde{\boldsymbol{\rho}}_{i}^{(l)}\right\|\left\|\tilde{\boldsymbol{\rho}}_{j}^{(l)}\right\|}\right)^{2}}
$$

\section{Discussion}

Some useful comments are provided here to get more insight onto the considered testing method and its potential applications and extensions.

- The asymptotic performance analysis of SOBI derived in [5], shows that the separation performance of two sources $s_{i}$ and $s_{j}$ depends on the angle between their respective correlation vectors $\widetilde{\boldsymbol{\rho}}_{i}$ and $\widetilde{\boldsymbol{\rho}}_{j}$. Hence, measuring this angle gives a hint on the interference rejection level of the two considered sources.

As a consequence, one can use the measure of this angle not only to test the separability of the two sources but also to guarantee a target (minimum) separation quality. Choosing the threshold $\alpha_{(i, j)}$ accordingly is an important issue currently under investigation.

- The testing method can be incorporated into a two stage separation procedure where the first stage consists in a second order decorrelation method (e.g. SOBI). The second stage would be an HOS-based separation method applied only when the testing indicates a failure of separation at the first step.

- In many practical situations, one might be interested by only one or few source signals. This is the case for example in the interference mitigation problem in [11] or in the power plants monitoring applications [12]. In this situation, the partial identifiability result is of high interest as it proves that the desired source signal can still be extracted even if a complete source separation cannot be achieved.

- We believe that similar testing procedure can be used for HOS-based BSS methods, at least for those like JADE [13], that are based on $4^{\text {th }}$ order decorrelation. This would be the focus of future research work.

\section{Simulation results}

We present in this section some simulation results to illustrate the performance of our testing method. In the simulated environment we consider uniform linear array with $n=2$ sensors receiving the signals from $m=2$ unit-power first order autoregressive sources (with coefficients $a_{1}=0.95 e^{j 0.5}$ and $a_{2}=0.5 e^{j 0.7}$ ) in the presence of stationary complex temporally white noise. The considered sources are separable according to the identifiability result, i.e. their respective correlation vectors $\widetilde{\boldsymbol{\rho}}_{1}$ and $\widetilde{\boldsymbol{\rho}}_{2}$ are linearly independent. The time lags (delays) implicitly involved are $\tau_{0}, \cdots, \tau_{9}$ (i.e., $K=10$ ). The signal to noise ratio (SNR) is defined 
as SNR $=-10 \log _{10} \sigma_{n}^{2}$, where $\sigma_{n}^{2}$ is the noise variance. We use SOBI algorithm [5] to obtain the decorrelated sources. The statistics in the curves are evaluated over 2000 Monte-Carlo runs. We present first in figure 1(a) a simulation example where we compare the rate of success of the testing procedure (success means that we decide the 2 sources have been separated) to detect the sources separability for different sample sizes versus the SNR in $\mathrm{dB}$. The confidence interval is fixed to $\beta=99.7 \%$. One can observe from this figure that the performance of the testing procedure degrades significantly for a small sample size due to the increased estimation errors and the fact that we use the asymptotic normality of considered statistics. In figure 1(b), we present a simulation example where we compare the rate of success according to the sample size for different confidence intervals. The SNR is set to $25 \mathrm{~dB}$. Clearly, the lower the confidence interval is, the higher is the rate of success of the testing procedure. Also, as observed in figure 1 , the rate of success increases rapidly when increasing the sample size. In figure 2(a), we present a simulation example where we plot the rate of success versus the confidence interval $\beta$ for different sample sizes and for $\mathrm{SNR}=25 \mathrm{~dB}$. This plot shows somehow the evolution of the rate of success w.r.t. the 'false alarm rate' and confirms the results of the two previous figures.

The simulation example presented in figure $2(\mathrm{~b})$ assumes two source signals with parameters $a_{1}=0.5 e^{j 0.5}$ and $a_{2}=0.5 e^{j(0.5+\delta \theta)}$, where $\delta \theta$ represents the spectral overlap of the two sources. The number of sensors is $n=5$, the sample size is $T=1000$ and the $\mathrm{SNR}=30 \mathrm{~dB}$. Figure $2(\mathrm{~b})$ shows the rate of success versus the spectral shift $\delta \theta$. As we can see, small values of $\delta \theta$ lead to high rates of 'nonseparability' decision by our testing procedure. Indeed, when $\delta \theta$ is close to zero the two vectors $\widetilde{\boldsymbol{\rho}}_{1}$ and $\widetilde{\boldsymbol{\rho}}_{2}$ are close to 'linear dependency'. That means that the separation quality of the two sources is poor in that case which explains the

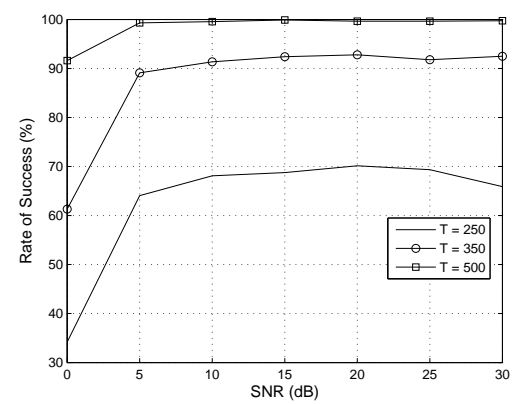

(a)

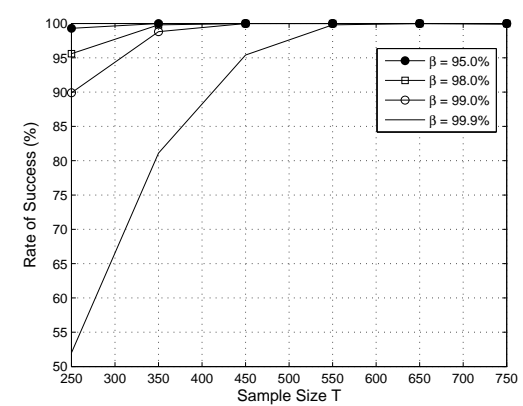

(b)

Fig. 1. (a)Rate of success versus SNR for 2 autoregressive sources and 2 sensors and $\beta=99.7 \%$ : comparison of the performance of our testing algorithm for different sample sizes T; (b)Rate of success versus sample size $T$ for 2 autoregressive sources and 2 sensors and $S N R=25 d B$ : comparison of the performance of our algorithm for different confidence interval $\beta$. 
observed testing results. In the last figure, we assume there exist three sources. The first two sources are complex white gaussian processes (hence $\widetilde{\boldsymbol{\rho}}_{1}=\widetilde{\boldsymbol{\rho}}_{2}$ ) and the third one is an autoregressive signal with coefficient $a_{3}=0.95 e^{j 0.5}$. The plots in figure 2(c) compares the average values of scalar products for $\widetilde{\boldsymbol{\rho}}_{i}$ and $\widetilde{\boldsymbol{\rho}}_{j}(i, j=1,2,3)$ with their corresponding threshold values $1-\alpha_{(i, j)}$ versus the SNR. The sample size is fixed to $T=500$ and the number of sensors is $n=3$. This example illustrates the situation where two of the sources (here sources 1 and 2) cannot be separated (this is confirmed by the testing result) while the third one is extracted correctly (the plots show clearly that $R(1,3)<1-\alpha_{(1,3)}$ and $\left.R(2,3)<1-\alpha_{(2,3)}\right)$.

\section{Conclusion}

This paper introduces a new method for testing the second order identifiability condition of the blind source separation problem. In simple words, this testing allows us to 'blindly' check, out of the observation, whether the unknown sources have been correctly separated or not. To evaluate the statistics needed for the testing procedure we used the jackknife (resampling) technique. The simulation results illustrate and assess the effectiveness of this testing procedure at least for moderate and large sample sizes.

\section{References}

1. A.K. Nandi (editor), "Blind estimation using higher-order statistics." Kluwer Acadimic Publishers, Boston 1999.

2. D. T. Pham and J. Cardoso, "Blind source separation of instantaneous mixtures of nonstationary sources," IEEE Trans. SP., Vol. 49, no. 9, pp. 1837-1848, 2001.

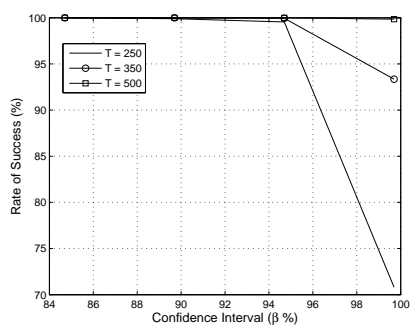

(a)

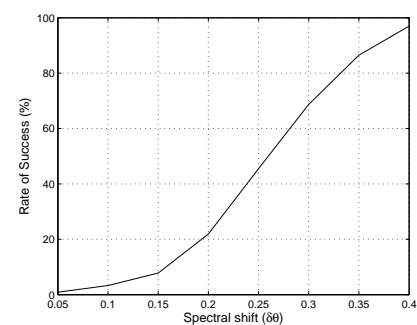

(b)

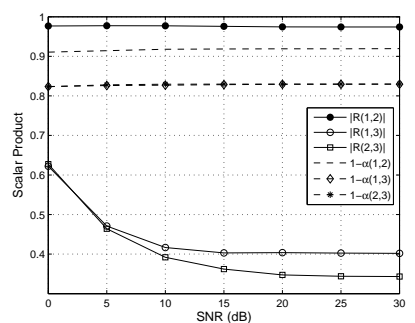

(c)

Fig. 2. (a)Rate of false alarm versus confidence interval $\beta$ for 2 autoregressive sources and 2 sensors and $S N R=25 \mathrm{~dB}$ : comparison of the performance of our algorithm for different sample size $T$; (b)Rate of success versus spectral shift $\delta \theta$ for 2 autoregressive sources and 5 sensors and $S N R=25 d B ;(c)$ Average values of the $|R(i, j)|$ and thresholds $1-\alpha_{(i, j)}$ versus SNR for 3 sources and 3 sensors : 2 sources are complex white gaussian processes and the third one is an autoregressive signal. 
3. K. Abed-Meraim, Y. Xiang, Y. Hua, "Generalized second order identifiability condition and relevant testing technique", In Proc. ICASSP, Vol. 5, 2989-2992, 2000.

4. L. Tong, R. Liu, V.C. Soon, Y.H. Huang, "Indeterminacy and identifiability of blind identification", IEEE-T-CS, Vol. 38, pp. 499-509, 1991.

5. A. Belouchrani, K. Abed-Meraim, J.F. Cardoso, E. Moulines, "Blind source separation using second order statistics", IEEE Trans. SP, pp. 434-444, 1997.

6. M. Kawamoto, K. Matsuoka, and M. Oya, "Blind separation of sources using temporal correlation of the observed signal", IEICE Tr. on Fundamentals of Electronics Communications and Computing E80A(4):695-704, Apr. 1997.

7. K. Abed-Meraim, Y. Hua, and A. Belouchrani, "A general framework for blind source separation using second order statistics", Eighth IEEE Digital Signal Processing Workshop, Utah, USA, (CD-ROM), Aug. 1998.

8. A.M. Zoubir and B. Boashash, "The bootstrap and its application in signal processing", IEEE Signal Processing Magazine, Vol. 15, pp. 56-76, 1998.

9. B. Efron, "The jackknife, the bootstrap and other resampling plans", CBMS Monograph 38, Society for Industrial and Applied Mathematics, Philadelphia, 1982.

10. R.G. Miller, "The jackknife - A review", Biometrika, Vol. 61, pp. 1-15, 1974.

11. A. Belouchrani, M.G. Amin, M.G. Chenshu Wang "Interference mitigation in spread spectrum communications using blind source separation," Asilomar Conference, pp. 718-719, 1996

12. G. D'Urso, P. Prieur, C. Vincent "Blind identification methods applied to Electricite de France's civil works and power plants monitoring," Higher-Order Statistics, 1997. Proceedings of the IEEE Signal Processing Workshop, pp. 82-86, 1997.

13. J.F. Cardoso and A. Souloumiac, "A.Blind beamforming for non-Gaussian signals", Radar and Signal Processing, IEE Proceedings F, Vol. 140, pp. 362-370, 1993. 